\title{
La Axiomatización de la Teoría del Derecho ${ }^{1}$
}

\author{
The Axiomatization of the Theory of Law
}

\author{
Juan de Dios González Ibarra \\ Universidad Autónoma del Estado de México (UAEM), México, México
}

José Luis Díaz Salazar

Centro De Investigación y Docencia en Humanidades del Estado de Morelos (CIDH), Morelos, México

\begin{abstract}
Resumen: Las dos ramas principales de la lógica son la lógica formal (o lógica menor) y la lógica material (o lógica mayor). La lógica material se ocupa del contenido semántico de la argumentación. La lógica formal se interesa por la forma o estructura de los razonamientos, es la lógica clásica propiamente dicha. Establecer cuáles son las formas correctas y válidas de los raciocinios, la verdad de las premisas y de las conclusiones es una preocupación derivada para esta rama.
\end{abstract}

Palabras clave: Axiomatización. Estructura Formal. Vocabulario. Reglas de Definición. Reglas de Inferencia.

\begin{abstract}
The two main branches of logic are formal logic (or logic low) and material logic (or logic high). The material logic deals with the semantic content of the argument. Formal logic is concerned with the form or structure of reasoning, classic logic is proper. Establish what the correct and valid forms of reasoning the truth of the premises and conclusions is a secondary concern for this branch.
\end{abstract}

Keywords: Axiomatization. Formal Structure. Vocabulary. Definition Rules. Rules of Inference.

\section{Introducción}

La formulación inicial de la lógica matemática (axiomatización) se debe fundamentalmente a Frege. Su gran aportación fue, inventar un sistema de símbolos mediante el cual los lógicos y los no tanto, pudieran formular las inferencias estudiadas por la lógica proposicional de Aristóteles, como aquellas a las que el método aristotélico no puede ser apli-

\footnotetext{
${ }^{1}$ Recebido em: 10/07/2013

Aprovado em: 19/08/2013
} 
cado. En la teoría del Derecho, las dificultades y malentendidos de interpretación y comunicación se originan por obviar sus reglas sintácticas, semánticas y pragmáticas. La función básicas de la axiomatización es la de minimizar el riesgo del uso inadecuado del lenguaje en el curso de los razonamientos lógico-deductivos normativos. Para evitar la aparición de antinomias, ${ }^{2}$ es necesario la especificación de la estructura formal y el vocabulario de un lenguaje. Para especificar la estructura de un lenguaje, tenemos que: a) determinar la clase de palabras y expresiones que van a ser utilizadas. b) indicar todas las palabras que utilizaremos sin definirlas (términos definidos o primitivos ${ }^{3}$ ) y c) las reglas de definición para introducir términos definidos o nuevos. Por último, formular las condiciones en que una oración del lenguaje puede aseverarse. Especialmente todos los axiomas (u oraciones primitivas), que decidamos afirmar sin necesidad de prueba; y dar las reglas de inferencia (o reglas de prueba) mediante las cuales se puedan deducir nuevas oraciones a partir de otras.

\section{Objeto, Finalidad y Método de la Teoría del Derecho Garan- tista}

Se corresponden lógicamente, conforme lo muestra Luigi Ferrajoli, apoyándose en la clasificación tripartita semiótica (FERRATER; LEBLANC, 1994, p. 17-19) ${ }^{4}$ del norteamericano Charles Morris (19011979), como son: a) la semántica (objeto sobre el que versa); b) la pragmática (finalidad u objetivo); y c) la sintáctica (método que utiliza para su

\footnotetext{
2 Tarski afirma que el lenguaje formal que se utilice para dar una definición de verdad debe cumplir con condiciones específicas y que es aconsejable ocuparse primero de las antinomias que directamente implican a la noción de verdad.

3 Términos definibles e indefinibles o primitivos: los definible, incluyen aquellas palabras que son definidas o explicadas dentro del campo especifico de conocimiento al que pertenecen. Los indefinibles, agrupan todas las palabras utilizadas por la ciencia o disciplina para realizar las definiciones o explicaciones de los primeros.

4 La semiótica como metalenguaje tiene tres dimensiones: la sintaxis, la semántica y la pragmática, la primera estudia las relaciones de los signos entre sí, la semántica "los signos en su relación con los objetos designados" y la pragmática "estudia los signos en su relación con los sujetos que los usan”.
} 
construcción) de la teoría (Morris, 1985, p. 18-20), ${ }^{5}$ bajo un status metateórico. (PALAU, 2002, p. 34)

La semántica, encargada del contenido empírico e informativo de la teoría en relación con el derecho positivo y del universo discursivo que crea en el imaginario social (DÍAZ, 1997, p. 11). ${ }^{6}$ La pragmática, dedicada a la finalidad explicativa de la teoría, su crítica y prospectiva que plantea respecto del derecho, sin salir del propio derecho. La sintáctica, dedicada a las relaciones entre los signos y del método axiomático utilizado para la formación de sus conceptos y proposiciones.

De tal manera, un lenguaje formal tendrá una doble dimensión, la estrictamente sintáctica, sin significado, pero cuyo análisis nos proporciona más elementos para conocer a la teoría original y, la dimensión semántica, en la cual se tiene el significado que se pretende dar a los símbolos cuyo estudio determina la teoría formal con que se construirá en el lenguaje formal dado. Ambas dimensiones se complementan y enriquecen recíprocamente.

Nuestro objetivo es la construcción de un modelo formal del pensamiento inductivo-deductivo. Omitiendo a la sintáctica, para Ferrajoli sumariamente la semántica y la pragmática es aquello de lo que habla y para lo que sirve una teoría del derecho. Dicho status metateórico, se refiere a la vinculación y relación entre la teoría y las aproximaciones disciplinarias (jurídica, sociológica y politológica) del estudio del derecho, así como del rol normativo y explicativo, teniendo en cuenta la estructura constitucional, la teoría del derecho y la ciencia jurídica en su dimensión rigurosa iuspositiva.

5 Dimensiones de teoría de los signos (semiosis) reconocidas por Charles Morris, (Foundations of the Theory of Signs, (1938), traducción al italiano de F. Rossi Landi, Lineamenti di una teoria dei segni, Paravia, Turín, 1954, al castellano de R. Grasa, Fundamentos de la teoría de los signos, Paidós, Barcelona, 1985.

6 La semántica se constituye a partir de las coincidencias valorativas de las personas, pero también de las resistencias. Se manifiesta en lo simbólico (lenguaje y valores) y en el accionar concreto entre las personas (prácticas sociales). 
Luego entonces la gran pregunta que nos orienta es ¿pero cuál es la objetivo de la lógica (GARCÍA MÁYNEZ, 2007, p. 7)7 que hemos desarrollado en la teoría derecho? Como una (pero no la respuesta) respuesta (CARROLL, 2002, p. 12-13) $)^{8}$ encontramos que es necesaria si lo que se pretende es construir tareas en las que interviene la capacidad inductiva-deductiva, donde se requiere: a) el dominio del discurso; b) razonar con tal conocimiento y c) guiar tal razonamiento (PARKINSON, 1965, p. 97). ${ }^{9}$ Además se busca disminuir los elementos subjetivos de incongruencia y la ambivalencia en la expresión del razonamiento silogístico normativo (ECHEVERRÍA, 1981, p. 130). Tal es el objetivo de la lógica que hemos estudiado en esta obra y concretamente del método axiomático aplicado al derecho.

El fundador del garantismo sostiene que si el lenguaje de la teoría del Derecho es un lenguaje artificial (FERRAJOLI, 2011, p. 67.), ${ }^{10}$ construido por el propio teórico, entonces no sólo es posible y oportuno, sino que es obligado construirlo de la manera más rigurosa posible. Por lo tanto, se realiza una reconstrucción del lenguaje teórico de la ciencia jurídica, en base a un lenguaje "artificial" o "convencional", elaborado por el teórico jurista. De ello resulta, una teoría "formal" que no nos dice absolutamente nada de la realidad jurídica, sin una adecuada interpretación semántica (COMANDUCCI, 1999, p. 132). Su gran aportación en el tercer tomo de Principia iuris es que podamos entender la sintaxis del derecho desde la metalógica del lenguaje simbólico.

Las ventajas del método axiomático son: a) la univocidad semántica de todos los términos teóricos asegurada por sus definiciones sobre la

\footnotetext{
7 Sostiene que "la lógica del derecho comprende tres partes: doctrina del juicio, doctrina del concepto y doctrina del raciocinio".

8 Nos señala "cuenta usted con mi promesa de que la lógica simbólica aparecerá ante usted como una de las más estimulantes recreaciones intelectuales, tal vez la más fascinante".

9 Leibniz fue el primero en afirmar la posible existencia de algo equivalente a una lógica formal completa para describir el razonamiento. Peano reconoció que Leibniz fue el verdadero fundador de la lógica matemática.

10 Paolo Comanducci se ha dedicado a exponer un modelo lingüístico, metaético y lógico del discurso y del razonamiento judicial.
} 
base de reglas de formación preestablecidas; b) la coherencia interna del discurso teórico, asegurada por las demostraciones de todas sus tesis no primitivas, o teoremas, sobre la base de las reglas de transformación (las de la lógica de predicados, la lógica de enunciados y la lógica modal) a su vez preestablecidas; c) la transparencia de las elecciones que están detrás de la teoría y que son todas ellas y únicamente expresadas por las asunciones (postulados y definiciones), siendo todas las otras tesis, respecto de aquéllas, lógicamente verdaderas y d) la fecundidad del método, que no es sólo un método de control, sino todavía antes un método de descubrimiento, dado que no todas las tesis válidamente demostrables como verdaderas son intuitivas y no todas las tesis intuitivas son válidamente demostrables como verdaderas.

Para Ferrajoli, el objeto de la teoría del derecho, es la identificación y análisis de las estructuras formales de los ordenamientos jurídicos. ${ }^{11}$ Describe los aspectos metodológicos de su teoría del derecho y el método axiomático como las relaciones sintácticas entre los signos de la teoría utilizado para la formación de sus conceptos y proposiciones. La lógica simbólica tiene por objeto de estudio el cálculo de la inferencia, para demostrar la validez de un argumento, a través de una serie de reglas. Para el cálculo proposicional en su aplicación es necesario la simbolización del lenguaje natural (lenguaje artificial), el empleo de conectivos lógicos y tablas de verdad. Un sistema formal no es simplemente una axiomática, es una abstracción mayor a partir de lo axiomático. ${ }^{12}$

Para la teoría axiomática del derecho es necesario formular un lenguaje construido a medida y necesidad. El lenguaje como un sistema simbólico complejo que sirve a la comunicación tiene tres aspectos o dimensiones: la sintáctica, la semántica y la pragmática. La dimensión sintáctica, es la relación que se establece entre los signos de un lenguaje. La sintaxis estudia las diversas combinaciones de signos que dan lugar a combinaciones de ellos con la propiedad de estar bien formadas. Por

11 Por su parte García Máynez señala en la obra citada: "A diferencia de la lógica pura que analiza la forma de los juicios...la del derecho es el estudio sistemático de la estructura de las normas, los conceptos y los razonamientos jurídicos".

12 En la axiomática es importante cuál es el axioma primario y su elección. En un sistema formal no, ya que es relativo y determinado por convención o comodidad. 
ejemplo, no es lo mismo afirmar congruentemente, no hay que afanarse en ser justo, sino parecerlo, que expresar incongruentemente "si no parecerlo afanarse en ser no hay", lo que demuestra la importancia de la construcción sintáctica. En los lenguajes artificiales ocurre algo pareci$\mathrm{do}^{13}$. La dimensión semántica se refiere a las relaciones de los signos con sus correspondientes significados (signos-significados). La semántica trata de investigar las relaciones de los signos con aquello que constituye su interpretación (aunque al margen de los contextos en que estos signos son usados por sus hablantes).

\section{Presentación Axiomática de la Lógica Proposicional}

El sistema axiomático, desde los tiempos de la geometría euclidiana, es la forma propia de presentar el cálculo o lenguaje formalizado. Lo particular del sistema axiomático radica en disponer de un conjunto de enunciados o fórmulas que se admiten sin demostración, a partir de los cuales se obtienen todas las demás afirmaciones de la teoría llamados teoremas. Las fórmulas aceptadas sin demostración se llaman axiomas o postulados. El conjunto de los axiomas más la definición de enunciado o fórmula del sistema y el conjunto de las reglas para la obtención de teoremas a partir de los axiomas (reglas de transformación) forman la base primitiva del sistema. ${ }^{14} \mathrm{El}$ nombre de "reglas de transformación" está justificado, porque las operaciones mediante las cuales se obtienen teoremas a partir de los axiomas consisten en transformaciones de éstos: a) como sustituciones de unas variables por otras y b) composición de axiomas para formar otras fórmulas.

\footnotetext{
13 Las gramáticas formales son sistemas de manipulación simbólica que permiten generar cadenas de símbolos, llamadas por esto bien formadas, o bien reconocer cuándo una cadena dada está, en efecto, bien formada.

14 Suele distinguirse entre sistemas axiomáticos formalizados y no formalizados. La diferencia principal consiste en que los formalizados presentan explícitamente todas las reglas de transformación, mientras que los otros no lo hacen. En un sistema axiomático formalizado el conjunto de los axiomas y el de las reglas de transformación son ambos efectivos.
} 
Un teorema, es una fórmula bien formada (fbf) que puede ser demostrada dentro de un sistema formal. La demostración, es el punto central en la lógica y la matemática. Un teorema requiere de un marco lógico, un conjunto de axiomas (sistema axiomático) y un proceso de inferencia. Tanto en lógica proposicional como en la de primer orden, cualquier afirmación demostrada, se nombra teorema. De forma más concreta, en lógica, una demostración, es una secuencia finita de fórmulas bien formada, $\mathrm{F}_{1}, \ldots, \mathrm{Fn}$, tales que cada $\mathrm{F}_{1}$ es o bien un axioma o bien un teorema que se sigue de dos fórmulas anteriores $F_{j}$ y $F_{k}$ (tales que $j<1$ y $k<1$ ) mediante una regla de deducción.

Tanto las reglas de transformación como de formación de fórmulas o enunciados son metalingüísticas respecto de las fórmulas del sistema, por ser afirmaciones de lo que puede hacerse con fórmulas del sistema. Igualmente, un enunciado que diga que tal o cual fórmula es un axioma, será metalingüístico respecto del lenguaje al que pertenezca dicho axioma. Finalmente, los teoremas se obtienen de los axiomas, también los predicados no primitivos, no contenidos en los axiomas se obtienen del sistema a partir de las nociones primitivas contenidas en los axiomas. El proceso se especifica mediante reglas de definición.

\section{Idea de Demostración}

Un sistema axiomático se organiza para formar con precisión la fundamentación de los teoremas de una teoría en sus axiomas y la demostración como el modo formal de fundamentar. Una demostración en un sistema axiomático, es una sucesión finita de fórmulas cada una de las cuales es o bien un axioma o bien una fórmula obtenida inmediatamente de un axioma por la aplicación de una regla de transformación, o bien una fórmula obtenida de otra $\mathrm{u}$ otras de los dos géneros anteriores mediante una aplicación de las reglas de transformación. Un teorema es en sentido estricto, una fórmula de cualquiera de las dos clases citadas y, en sentido amplio, es cualquier fórmula fundamentada del sistema. (SACRISTÁN, 1969, p. 103-104) 


\section{Sistema Axiomático de Principia Mathematica de Russell/Whi- tehead}

I. Símbolos primitivos

1. Variables proposicionales: $\mathrm{p}, \mathrm{q}, \mathrm{r}, \mathrm{s}$

2. Operadores: $\sim, \vee$

3. Signos de agrupación: '( )', '[ ]', ' \{\} '

II. Reglas de formación:

1. Toda variable proposicional es una fórmula bien formada (fbf).

2. Si P es una fbf, entonces $\sim \mathrm{P}$ también lo es.

3. Si $\mathrm{P}$ y $\mathrm{Q}$ son $\mathrm{fbfs}$, entonces $\mathrm{P} \vee \mathrm{Q}$ también lo es.

4. Éstas son todas las reglas de formación del sistema.

III. Definiciones:

1. Definición 1 (Def. 1) $\mathrm{P} \rightarrow \mathrm{Q}=$ def. $\sim \mathrm{P} \vee \mathrm{Q}$

2. Definición 2 (Def. 2) $\mathrm{P} \wedge \mathrm{Q}=$ def. $\sim(\sim \mathrm{P} \vee \sim \mathrm{Q})$

3. Definición 3 (Def. 3) $\mathrm{P} \leftrightarrow \mathrm{Q}=\operatorname{def} .(\mathrm{P} \rightarrow \mathrm{Q}) \wedge(\mathrm{Q} \rightarrow \mathrm{P})$

IV. Reglas de transformación:

2. Regla de sustitución (R.1.)

En una fórmula cualquiera toda variable proposicional puede ser sustituida por cualquier fbf, siempre que la sustitución se verifique en todos los lugares en que dicha variable aparezca.

2. Regla de separación (R.2.)

Si P es una fórmula derivable del sistema y también lo es la fórmula

$\mathrm{P} \rightarrow \mathrm{Q}$, entonces

Q es otra fórmula derivable. 
V. Axiomas:

$$
\begin{array}{ll}
\text { Ax. } 1 & (\mathrm{p} \vee \mathrm{p}) \rightarrow \mathrm{p} \\
\text { Ax } 2 & \mathrm{q} \rightarrow(\mathrm{p} \vee \mathrm{q}) \\
\text { Ax } 3 & (\mathrm{p} \vee \mathrm{q}) \rightarrow(\mathrm{q} \vee \mathrm{p}) \\
\text { Ax } 4 & (\mathrm{q} \rightarrow \mathrm{r}) \rightarrow[(\mathrm{p} \vee \mathrm{q}) \rightarrow(\mathrm{p} \vee \mathrm{r})]
\end{array}
$$

\section{Lógica Proposicional}

La lógica de proposiciones (DOWEK, 2001, p. 17) ${ }^{15}$ es la parte más elemental de la lógica moderna o matemática. Sólo se examinan las relaciones lógicas existentes entre proposiciones consideradas como un todo. Dispone de medios de análisis formal llamadas inferencias (lenguaje simbólico y métodos específicos) y la validez de éstas se determina por las relaciones entre proposiciones.

\subsection{Noción de Proposición}

El lenguaje, en sentido estricto, es un sistema convencional de signos. Sirve para afirmar o negar (oraciones aseverativas o declarativas); expresar deseos (oraciones desiderativas); formular preguntas (oraciones interrogativas); expresar sorpresa o admiración (oraciones exclamativas o admirativas) e indicar exhortación, mandato o prohibición (oraciones exhortativas o imperativas).

Ejemplo:

“a)" y "b)" son proposiciones, porque tiene sentido decir que "a)" es verdadera y que "b)" es falsa. En consecuencia, la verdad y la falsedad son sus propiedades, sólo las proposiciones pueden ser verdaderas o falsas.

Para que una expresión lingüística sea proposición debe cumplir los siguientes requisitos:

1) Ser oración.

15 Afirma que "Con los estoicos aparece la noción de proposición formada con la contribución de conjunciones". 
2) Ser oración aseverativa, $y$

3) Ser o bien verdadera o bien falsa.

\subsection{Proposición, Oración y Enunciado}

Es preciso diferenciar una proposición (SCHMILL, 2003, p. 51) $)^{16}$ (objeto conceptual o constructo) de las oraciones (objetos lingüísticos) que la designan, expresan o formulan, así como distinguir una oración de sus diversas enunciaciones ${ }^{17}$ (acto psicofísico) orales, escritas, o ademanes. La enunciación y la percepción de una oración son procesos y objetos físicos en sentido amplio. No así la oración misma, ésta puede considerarse una clase de enunciaciones concretas, en circunstancias particulares. Por ejemplo, las oraciones " $5>2$ ", "VI > II", "Five is greater than two" $\mathrm{y}$ "Cinco es mayor que dos" expresan o designan una misma proposición.

Pero hay oraciones gramaticales que no formulan proposición alguna, ejemplo "el número cinco aleteó" y "la raíz cuadrada de una melodía es igual a un sueño". (BUNGE, 1982, p. 62-65)

Las proposiciones vinculan clases entre sí. Una clase es la denotación o extensión de un término, es el conjunto de individuos u objetos a los que se aplica una misma palabra o expresión. Cuando las proposiciones relacionan clases claramente y sin condiciones, son proposiciones categóricas y muestran qué tipo de relación tiene una clase con otra. La proposición, todos los hombres son mortales vincula la clase, hombres con la clase mortales y la proposición Sócrates es mortal, vincula la clase, de un solo miembro (Sócrates), con la clase de los mortales. La conexión entre clases, informa de los hechos en una proposición.

Las clases relacionadas por una proposición, de acuerdo con su ubicación en ésta toman el nombre de sujeto o de predicado. El sujeto es aquello de lo que la proposición dice algo, al que se califica o indica que posee una propiedad; en tanto el predicado, lo que se dice en relación al sujeto. De tal forma, en la proposición algunas leyes son vigentes, el su-

\footnotetext{
16 El lenguaje L "sólo comprende el cálculo proposicional".

17 Enunciar, escuchar, escribir o leer una oración, por ejemplo si expresamos verbalmente "cinco es mayor que dos" se ejecuta un acto psicofísico.
} 
jeto es leyes y el predicado vigentes. Tanto al sujeto como al predicado se les denomina términos de la proposición y a la palabra que los une copula y su calidad (de la proposición) es su sentido afirmativo o negativo. El primero cuando incluye una clase en otra, negativo cuando la excluye. La cantidad de una proposición es la medida en que se incluye o excluye una clase en otra, universal si es de forma total y particular si es parcial. La cantidad de una proporción la determinan palabras denominadas cuantificadores como todos, algunos y ninguno.

Las relaciones entre clases de una proposición atendiendo a su calidad y cantidad son de los siguientes tipos:

a) Una clase se encuentra totalmente incluida en otra. Citemos algunos ejemplos: Todos los derechos humanos son parte de la Constitución Mexicana, es una proposición universal afirmativa. Emilio Álvarez Icaza Longoria es Secretario Ejecutivo de la Comisión Interamericana de Derechos Humanos, es una proposición singular afirmativa.

b) Una clase se encuentra incluida de forma parcial en otra. Ejemplos: algunos de los derechos humanos son negativos (VELÁZQUEZ, 2006, p. 76), ${ }^{18}$ es una proposición particular afirmativa. Algunas actividades positivas son impuestas al Estado por el bloque de constitucionalidad vigente ${ }^{19}$ relacionados con los derechos humanos, es una proposición particular afirmativa.

c) Una clase se encuentra excluida totalmente de la otra. Ejemplos: ninguna proposición constituye de suyo un axioma, es una proposición universal negativa. Francisco Zuazo no está obligado a lo imposible, es una proposición singular negativa.

\footnotetext{
18 Un grupo grande de derechos llamados "derechos negativos", se distingue (como grupo) por el hecho de que sus miembros se definen por completo en términos de las obligaciones que otros tienen de no inferir en ciertas actividades de la persona que tiene el derecho dado.

19 Los derechos positivos no hacen más que imponer obligaciones negativas. También implican que algunos otros agentes (no siempre está claro quién) tiene una obligación positiva de proporcionar a los poseedores del derecho lo que necesitan para buscar sus intereses.
} 
d) Una clase es parcialmente excluida de otra. Ejemplos: algunas leyes no son constitucionales, es una proposición particular negativa.

La distribución de los términos de una proposición nos participa sobre la totalidad o parcialidad de los miembros de una clase, sea una clase manifestada por el sujeto o por el predicado. Hay distribución del término, si la información es sobre la totalidad de los miembros relacionados con ese término, no existe distribución, si la información es parcial.

\subsection{Características de los Enunciados}

El lenguaje cumple distintas funciones. Con el lenguaje interrogativo formulamos preguntas, con el lenguaje directivo damos órdenes, con el expresivo comunicamos distintos estados de ánimo, El lenguaje en que se manifiesta el conocimiento científico recibe el nombre de informativo, dicho lenguaje está formado por enunciados. Utilizamos esta expresión como sinónimo de proposición o de afirmación. La característica principal de los enunciados y, que los hacen aptos para volcar en ellos al conocimiento científico, es que pueden ser verdaderos o falsos.

\subsection{Validez o Corrección Formal de los Argumentos}

Debemos recordar en lo sucesivo que sólo los enunciados son verdaderos o falsos. Por diferencia no diremos que un argumento es verdadero o falso, sino diremos que es válido o inválido. La validez es la relación objetiva de correspondencia entre las premisas y la conclusión. La validez del razonamiento es independiente de la verdad o falsedad de las premisas, ya que puede existir un razonamiento valido con premisas falsas.

Ejemplo: mañana es 30 de febrero, es un argumento inválido, ya que sabemos que ningún mes de febrero tiene 30 días. La lógica se ocupa primeramente por establecer una clara distinción entre razonamientos válidos y razonamientos inválidos. Los razonamientos válidos son aquellos en los que la inferencia entre las premisas y la conclusión es perfecta. 
Lo esencial para determinar si un argumento es o no válido es analizar su forma o estructura (independientemente de su contenido material). Tres formas equivalentes de establecer el criterio de validez:

a) Si las premisas de un argumento válido son verdaderas, entonces su conclusión también es verdadera.

b) Es imposible que la conclusión de un argumento válido sea falsa siendo sus premisas verdaderas.

c) En un argumento válido, la verdad de las premisas es incompatible con la falsedad de la conclusión.

\subsection{Verdad o Adecuación Material de los Enunciados}

Se dice que un enunciado (o una proposición) es verdadero, cuando hay una correspondencia entre la realidad y el enunciado ${ }^{20}$. Si se afirma: la nieve es blanca, lo normal es que se tengan los medios para establecer su verdad o falsedad. Bajo qué condiciones esta oración es verdadera o falsa. Si nos basamos en la concepción clásica de la verdad, tendremos que afirmar que la oración es verdadera si la nieve es blanca y es falsa si la nieve no es blanca. La definición de verdad implica la siguiente equivalencia: La oración la nieve es blanca es verdadera si, y sólo sí, la nieve es blanca. ${ }^{21}$

\section{Solidez o Corrección Formal Unida a Adecuación Material}

Un argumento es sólido cuando es a la vez formalmente válido y materialmente adecuado (sus premisas y su conclusión son verdaderas). Dada lo anterior, la lógica puede ser pensada como el estudio de conjun-

\footnotetext{
20 A la verdad entendida como adecuación del intelecto a la cosa. Los medievales la denominan verdad lógica conforme con el gran teólogo de la iglesia católica Santo Tomas de Aquino, quien logró unir la filosofía griega con su teología de muchos dioses con lo expresado en la Biblia que sólo reconoce a un Dios único, infinito y omnipresente.

21 La frase la nieve es blanca aparece a la izquierda de esta equivalencia en letra cursiva, mientras que a la derecha aparece sin ella. A la derecha tenemos la oración y a la izquierda el nombre de la oración. Utilizando la terminología lógica medieval, las palabras "la nieve es blanca" aparecen en suppositio formalis y a la izquierda en suppositio materialis.
} 
tos consistentes de enunciados. Pero la palabra "consistencia" en lógica tiene un significado muy preciso. Cuando en lógica decimos, un conjunto de enunciados es consistente estamos afirmando que los enunciados del conjunto son compatibles entre sí. En otras palabras, que es posible para todos los enunciados del conjunto ser verdaderos al mismo tiempo en alguna situación.

Pondremos el clásico ejemplo de los estudiantes:

Yo sé todo lo que tengo que saber para acreditar los exámenes; todo lo he entendido y aprendido; pero en todos los exámenes no he tenido suerte y por eso los he reprobado todos.

Los anteriores enunciados forman un conjunto consistente. Es posible (aunque improbable) que sean todos verdaderos.

\section{Géneros de Proposiciones}

Existen dos clases: atómicas y moleculares. Las proposiciones atómicas (simples o elementales) carecen de conjunciones gramaticales típicas o conectivas ("y", "o", "si... entonces", "si y sólo si") o del adverbio de negación "no".

Ejemplos:

a) El hospital de Jesús es el dispensario más antiguo de América. $^{22}$

b) La lógica es diferente a la matemática.

Las proposiciones atómicas de acuerdo a sus elementos constitutivos pueden clasificarse en predicativas y relacionales.

Las proposiciones predicativas constan de sujeto y predicado.

Ejemplos:

22 Fue mandado construir por Hernán Cortés, al término de la conquista de la Gran Tenochtitlan en 1521, ahí reposan sus restos mortales y durante muchos años estuvo bajo el cuidado del genio mexicano ex rector de la UNAM el mexiquense Dr. Gustavo Baz Prada. 
a) El número 8 es par.

b) La gravedad es relativa.

Las proposiciones relacionales, vinculas dos o más sujetos entre sí. Ejemplos:

a) Ana es hermana de Roberto.

b) 5 es menor que 8 .

Las proposiciones moleculares (compuestas o coligativas), tienen alguna conjunción gramatical distintiva o conectiva o el adverbio negativo 'no'.

Ejemplos:

a) La lógica y la matemática son disciplinas formales.

b) El espacio es absoluto o es relativo.

c) Si dos ángulos adyacentes forman un par lineal, entonces son suplementarios.

d) Este número es impar si y sólo sí es divisible por dos.

\subsection{Proposiciones Moleculares}

Las proposiciones moleculares, según el tipo de conjunción gramatical $^{23}$ que las une, se clasifican en conjuntivas, disyuntivas, condicionales y bicondicionales, si tienen el adverbio "no" son negativas. Las proposiciones conjuntivas llevan la conjunción copulativa "y", o sus expresiones equivalentes "pero", "aunque", "aun cuando", "tanto... como...", "sino", "ni... ni", "sin embargo", "además”, etc.

Ejemplos:

a) El número tres es impar, pero el número cuatro es par.

b) Enrique es valiente, sin embargo es tonto.

c) Tanto uno como el otro son responsables.

23 La conjunción es una parte invariable de la lengua que se utiliza para enlazar oraciones y establecer relaciones de jerarquía entre ellas, explicar el tipo de relación semántica o de significado que existe entre ellas. 
d) Gabriel y Rafael son arcángeles.

e) "De" es una preposición y "El” es un artículo.

f) La materia no se crea ni se destruye.

g) Iré a cine aunque llueva.

Es importante aclarar, que en las proposiciones conjuntivas no es necesario que sus proposiciones componentes estén vinculadas en cuanto a su contenido; resulta suficiente la presencia de la conjunción " $y$ ". Una proposición conjuntiva es conmutativa, es decir, puede cambiar el orden de sus proposiciones componentes sin perturbar la conjunción. Esto es posible en la lógica, más no en el lenguaje natural. Por ejemplo, La proposición "Juan asesinó y huyó" no significa lo mismo que "Juan huyó y asesinó”. En el lenguaje natural, la primera sugiere una relación de causalidad, en cambio la segunda no. Sin embargo, desde el punto de vista lógico, las dos proposiciones conjuntivas son equivalentes.

Las pseudoproposiciones conjuntivas, son proposiciones que se muestran como proposiciones conjuntivas, en realidad son proposiciones atómicas relacionales. La ' $\mathrm{y}$ ', de los siguientes ejemplos, tiene carácter de término relacional y no propiamente de conjunción copulativa o conectiva.

Ejemplos:

a) Pedro y Juan son cuñados.

b) Pedro y Juan son apóstoles.

c) Pedro y Juan son socios.

d) Pedro y Juan son prófugos.

e) Pedro y Juan son huérfanos.

f) Pedro y Juan son colegas.

g) Pedro y Juan son mellizos.

h) Pedro y Juan son hermanos.

Las proposiciones disyuntivas llevan la conjunción disyuntiva "o", o sus expresiones equivalentes como "u", "y/o", etc. En el idioma español, la disyunción "o" tiene dos sentidos: uno inclusivo o débil y otro exclusivo o fuerte. La proposición disyuntiva inclusiva acepta que las dos 
alternativas se den conjuntamente. La proposición disyuntiva exclusiva no admite que las dos alternativas se den conjuntamente.

Ejemplos:

a) Juan es sobrino o es tío.

b) Ana está enferma o está sana.

c) Roberto es estudiante o es maestro.

d) Alicia es soltera o es casada.

Congruente con lo anterior "a)" y "c)", son proposiciones disyuntivas inclusivas o débiles porque en ellas no se excluye la posibilidad de que Juan pueda ser al mismo tiempo sobrino y tío, o de que Roberto sea estudiante y maestro a la vez; en cambio "b) y “d)" son proposiciones disyuntivas exclusivas o fuertes, porque en ellas se excluye la posibilidad de que Ana pueda estar enferma y sana al mismo tiempo y que Alicia sea soltera y casada a la vez.

En español no existe un signo especial para la disyunción inclusiva y exclusiva, en ambos casos se usa la misma partícula 'o'; pero en lógica sí existen signos especiales para distinguirlas.

Las proposiciones condicionales, conllevan la conjunción condicional compuesta "si... entonces...", o sus expresiones equivalentes como "si", "siempre que", "con tal que", "puesto que", "ya que", "porque", "cuando", "de", "a menos que", "a no ser que", "salvo que”, "sólo si”, "solamente si".

Ejemplos:

a) Si es viejo, entonces es débil.

b) Es carnívoro si se alimenta de carne.

c) El número uno es indivisible puesto que es la unidad.

d) Se llama isósceles siempre que el triángulo tenga dos lados iguales.

e) Cuando sane Pedro jugaremos futbol.

f) De mejorar el clima iremos al mar. 
Toda proposición condicional consta de dos elementos: antecedente y consecuente. La proposición que sigue a la palabra "si" es el antecedente y la que sigue a la palabra "entonces" será el consecuente. Toda proposición implicativa es condicional, pero no toda proposición condicional es implicativa. Únicamente, las proposiciones condicionales que son tautologías son implicativas.

Para que una proposición condicional sea lógicamente correcta, no es necesario que haya atingencia ${ }^{24}$ entre el antecedente y el consecuente, es decir, la verdad en una proposición condicional es independiente de las relaciones que puedan existir o no entre los significados del antecedente y el consecuente. Por ejemplo, la proposición "Si la luna gira alrededor de la tierra, entonces El Barcelona es campeón de España” es verdadera no obstante no tener relación alguna entre los significados de sus proposiciones componentes. Concluyendo, en toda proposición condicional el consecuente es condición necesaria del antecedente y el antecedente es condición suficiente del consecuente. Por ejemplo, en la proposición condicional "si los cuerpos se calientan, entonces se dilatan", el consecuente "se dilatan" es condición necesaria del antecedente "se calientan" y el antecedente "se calientan" es condición suficiente del consecuente "se dilatan".

Las proposiciones bicondicionales llevan la conjunción compuesta “... sí y sólo si...", o expresiones equivalentes como "cuando y sólo cuando", “si..., entonces y sólo entonces...”, etc. Ejemplos:

a) Es fundamentalista si y sólo si es talibán.

b) Habrá producción cuando y sólo cuando se trabaje.

c) Si apruebo el examen de admisión, entonces y sólo entonces ingresaré a la facultad de medicina.

Las proposiciones bicondicionales, se caracterizan por establecer dos condicionales, de sentido inverso. Ejemplo, la proposición bicondicional "el triángulo es equilátero si y sólo si tiene tres lados iguales", establece dos condicionales de sentido inverso: "si es triángulo equilátero, entonces tiene tres lados iguales" y "si el triángulo tiene tres lados igua-

24 Conexión, relación, incumbencia. 
les, entonces es equilátero". En toda proposición bicondicional, el antecedente es condición necesaria y suficiente del consecuente y el consecuente es condición necesaria y suficiente del antecedente.

Las proposiciones negativas llevan el adverbio de negación 'no', o sus expresiones equivalentes: 'nunca', 'jamás', 'tampoco', 'no es verdad que', 'no es cierto que', 'es falso que', 'le falta', 'carece de', 'sin', etc. Ejemplos:

a) Nunca he interpretado esa música.

b) Jamás he visto al implicado.

c) Es imposible que el átomo sea molécula.

d) Es falso que el juez sea fiscal.

e) Al papá de Carlos le falta carácter.

\section{Cálculo de Predicados}

Es un sistema formal diseñado para estudiar la inferencia en los lenguajes de primer orden. Los lenguajes de primer orden son, a su vez, lenguajes formales con cuantificadores que alcanzan sólo a variables de individuo y con predicados y funciones cuyos argumentos son sólo constantes o variables de individuo (SIMON, 2009, p. 637). Nuestro objetivo ahora es comprobar que lo que hace válida una argumentación es su forma. ¿Pero cuáles son las dificultades de la lógica proposicional? Razonemos sobre lo siguiente:

Todos los hombres son mortales;

Todos los griegos son hombres;

Todos los griegos son mortales. 
Intuitivamente suponíamos éste como ejemplo de argumento correcto, pero, si lo simbolizamos en el contexto de la lógica de enunciados:

$$
p, q \therefore r
$$

No es un argumento correcto en virtud de su forma. Su validez no depende de las relaciones entre las premisas y la conclusión, sino de las relaciones entre partes de los enunciados.

Todos los A's son B's;

Todos los C's son A's;

$\therefore$ Todos los C's son B's.

Tomemos en cuenta de dos cosas:

1. El uso de símbolos para representar partes de un enunciado simple, implica un lenguaje formal más rico.

2. La naturaleza general de los enunciados "Todos los $A$ 's son $B$ 's", los hace enunciados moleculares o compuestos, por lo tanto, se necesitan cuantificadores.

De la formalización y el estudio de estructuras deductivas de este tipo se ocupa la lógica de predicados o de términos.

Empecemos por conceptualizar: Nombres, functores y relatores

a) Designador: una o varias palabras que hacen referencia a objetos o individuos. Forman el sujeto de una oración, los más usuales son los nombres. ${ }^{25}$

b) Constantes: en lugar de nombres del lenguaje ordinario utilizaremos las primeras letras minúsculas del alfabeto: a, b y c.

Emplearemos variables cuando queramos decir algo general. En el lenguaje ordinario, los pronombres juegan el papel de las variables en las fórmulas matemáticas. Las variables no designan a ningún objeto o individuo en particular. Como variables usaremos las útimas letras minúscu-

25 Los nombres son designadores simples, pero no todos los designadores son así. Por ejemplo: "La capital de Francia", es un designador compuesto. 
las del alfabeto: x, y y z. También utilizaremos variables cuando queramos decir algo general. En el lenguaje ordinario, los pronombres juegan el papel de las variables en las fórmulas matemáticas.

"Él ha sido el asesino" equivale a: "x ha sido el asesino";

"Yo he ido a trabajar" equivale a: "x ha ido a trabajar".

Las variables no designan a ningún objeto o individuo en particular.

a) Functores: son expresiones que seguidas de un número determinado de designadores, forman a su vez un designador. Usaremos, en vez de functores del lenguaje ordinario, los símbolos: $\mathrm{f}$, $\mathrm{g}, \mathrm{h}, \ldots$ Los functores podrán contener variables. Un functor que contiene variables no designa a ningún objeto o individuo, es decir, no es un designador: término abierto.

b) Relatores: Unidos a un número determinado de designadores forman un enunciado. Serán los enunciados atómicos de nuestro formalismo. Los relatores designan relaciones. Los relatores o predicados se usan para definir conjuntos (clases), cuando empleamos variables junto con dichos predicados. Emplearemos letras mayúsculas del alfabeto, "P”, “Q”, "R" para representar los relatores del lenguaje ordinario. Si en un enunciado sustituimos un designador por una variable, el resultado es lo que llamaremos una fórmula abierta.

Cuantificadores

a) Generalizador: son las partículas “todo" "cada", o "el”. Ejemplo la formalización del enunciado "Todos los hombres son mortales":

1. Para todo $\mathrm{x}$, si $\mathrm{x}$ es un hombre entonces $\mathrm{x}$ es mortal.

2. Para todo $x,(H(x) \rightarrow M(x))$.

donde "H(x)" simboliza " $\mathrm{x}$ es un hombre" $\mathrm{y}$ "M(x)" simboliza " $\mathrm{x}$ es mortal".

3. $(\forall \mathrm{x})(\mathrm{H}(\mathrm{x}) \rightarrow \mathrm{M}(\mathrm{x}))$. 
donde " $\forall$ " denota el cuantificador universal "para todo" y $x$ es la variable cuantificada. La variable " $x$ " en la fórmula $(\mathrm{H}(\mathrm{x}) \rightarrow \mathrm{M}(\mathrm{x}))$ se está ligada por el cuantificador.

b) Particularizador: son las partículas "alguno", también "existe", en "existe un número natural mayor que otro dado", o "algún", en "algún x es y", o "unos", en "unos perros son más fieles que otros", o "tiene" en "Moisés tiene un progenitor que le ama".

Pondremos como ejemplo la formalización del enunciado "algún animal es racional":

1. Existe un $x, x$ es animal y $x$ es racional.

2. Existe un $x,(A(x) \wedge R(x))$. donde " $A(x)$ " simboliza " $x$ es animal" $\mathrm{y}$ " $R(x)$ " simboliza " $x$ es racional".

3. $(\exists x)\left(A(x)^{\wedge} R(x)\right)$.

donde “ $\exists$ " denota el cuantificador existencial "existe un" y $x$ es la variable cuantificada.

c) Observaciones:

1. A partir de fórmulas abiertas podemos construir enunciados, anteponiendo una sucesión de cuantificadores con sus respectivas variables cuantificadas. Ejemplo: de la fórmula abierta " $\mathrm{R}(\mathrm{x}, \mathrm{y})$ " puede obtenerse el enunciado “ $(\forall \mathrm{x})(\exists \mathrm{y}) \mathrm{R}(\mathrm{x}, \mathrm{y})$ ”.

2. Entre el cuantificador universal y el cuantificador existencial, existe una semejanza funcional. Si tenemos un conjunto finito $\{\mathrm{a}, \mathrm{b}, \mathrm{c}\}$, el enunciado “ $(\forall x) R(\mathrm{x})$ " equivale a “ $R(a) \wedge R(b) \wedge R(c)$ " y “ $(\forall x) R(x)$ " equivale a " $R(a) \vee R(b)$ $\vee R(c) "$.

3. Existe relación entre los cuantificadores: “ $\neg(\forall \mathrm{x}) \neg$ ” es equivalente a " $(\exists x)$ ". 


\section{Afinidad entre los Principios Universales de la Lógica y la Ló- gica Jurídica}

Dado el objeto de la lógica, ésta se constituye en una poderosa herramienta para la obtención de las más sólidas seguridades dentro de las finalidades en la aplicación del derecho. Partiendo de los postulados teóricos de la lógica, se despliegan dichos axiomas, como elementos trascendentales de producción e interpretación de todo el conocimiento no solamente básico sino aplicado. Dicha aplicación para la obtención de razonamientos lógicos jurídicos se basa en los principios que hacen posible todo pensar lógico dirigido a la acción.

Nuestro querido maestro Eduardo García Máynez, desarrolló lo que llamó "principios apriorísticos del Derecho" que según él, deben ser aceptados como auténticos axiomas (GARCÍA MÁYNEZ, 1959, p. 5) antes de cualquier proceso jurídico y como requisito de validez del mismo, mismos que sirven de base a la ontología formal del Derecho:

1) Principio de identidad:

Un concepto idea u objeto son siempre idénticos a sí mismos. Su naturaleza (sustancia) no varía en el tiempo. Traza la equivalencia que puede existir entre un concepto y sus elementos constitutivos (hombre $=$ animal y racional). Emana su verdad y validez objetiva de la estructura ontológica de los objetos (ser iguales a sí mismos).

Identidad del imperativo: Todo mandato en que lo mandado es exactamente idéntico a lo que se está realizando, es necesariamente obedecido. Identidad de lo lógico jurídico: La norma que permite lo que no está jurídicamente prohibido o prohíbe lo que no está jurídicamente permitido, es necesariamente válida.

2) Principio de Contradicción:

Dos juicios contradictorios no pueden ser a un mismo tiempo verdaderos. Contradicción del imperativo: El mandato sería contradictorio si al mismo tiempo manda 
hacer y no hacer algo por lo tanto no pueden ser obedecidos ni válidos.

Contradicción de lo lógico jurídico: Dos normas se oponen contradictoriamente cuando teniendo ámbitos iguales de validez material, espacial y temporal, una permite y la otra prohíbe a los mismos sujetos la misma conducta.

3) Principio del tercero excluido:

Dos juicios contradictorios no pueden a un mismo tiempo ser falsos. Uno de los dos debe ser verdadero y el otro falso.

Tercero excluido del imperativo:

Dos mandatos contradictorios no pueden ser ambos desobedecidos. Uno de los dos debe ser obedecido no existe un tercer mandato.

Tercero excluido de lo lógico jurídico:

Dos normas de derecho contradictorias no pueden ser al mismo tiempo inválidas o inaplicables, una de las dos debe ser válida. Se excluye la posibilidad de una tercera norma válida en medio de dos inválidas.

4) Principio de razón suficientes:

Todo tiene su razón de ser.

Razón suficiente del imperativo: La razón de ser suficiente de cualquier imperativo jurídico y de todo el derecho es la conducta humana, cuya regulación de acuerdo a cierto valor y fines es el objetivo del orden jurídico y también su principio.

Razón suficiente en lo lógico jurídico:

Es un principio lógico y ontológico: lógico, la razón suficiente de las normas de derecho reside en la norma primaria.

El objeto de la lógica jurídica es el tratamiento cognoscitivo de las categorías del conocimiento lógico-jurídico que son fundamentalmente: la 
norma jurídica, el concepto jurídico, el juicio jurídico, la naturaleza coimplicante de la imputación jurídica, la inferencia jurídica, la argumentación jurídica (MANS, 1978, p. 134). Como hemos expuesto, la lógica jurídica es la aplicación de todos los recursos de la lógica al campo del derecho, sin embargo, nuestro interés en este espacio particular, es en concreto su aplicación a los razonamientos inválidos y validos en el derecho.

\section{Referencias}

BUNGE, Mario, Epistemología. La Habana: Ciencias Sociales, 1982. CARROLL, Lewis. El juego de la lógica. México: Grupo Editorial Tomo, 2002.

MORRIS, Charles. Fundamentos de la teoría de los signos. Barcelona: Paidós, 1985.

COMANDUCCI, Paolo. Razonamiento jurídico: elementos para un modelo. México: Distribuciones Fontamara, 1999.

DÍAZ, E. ¿Qué es el imaginario social? La Ciencia y el Imaginario Social, Bs. As., Biblos, 1997.

DOWEK, Gilles. La lógica. México: Siglo XXI editores, 2001.

ECHEVERRÍA, Javier. Leibniz. Barcelona: Barcanova, 1981.

FERRATER MORA, José; LEBLANC, Hugues. Lógica matemática. México: FCE, 1994.

GARCÍA MÁYNEZ, Eduardo. Ensayos filosóficos jurídicos. Xalapa: UV, 1959.

. Lógica del raciocinio jurídico. México: Fontamara, 2007.

. Los principios de la ontología formal del Derecho y su expresión simbólica. México: Imprenta Universitaria, 1953.

MANS PUIGARNAU, Jaime. Lógica para juristas. España: Bosch, 1978. 
PALAU, Gladys. Introducción a las lógicas no clásicas. Barcelona: Gedisa, UBAS, 2002.

PARKINSON, G. Logic and Reality in Leibniz's metaphysics. Oxford: Clarendon Press, 1965.

SACRISTÁN, Manuel. Introducción a la lógica y al análisis formal. Barcelona: Ariel, 1969.

SCHMILL, Ulises. Lógica y derecho. México: Fontamara, 2003. SIMON, Blackburn. The Oxford Dictionary of Philosophy, Oxford: Oxford University Press, 2009.

VELÁSQUEZ, Manuel, G. Ética de los negocios, conceptos y casos. México: Pearson Educación, 2006. 


\section{Anexo A - Símbolos Matemáticos y Lógicos}

$\begin{array}{ll}\sim & \text { Aproximadamente } \\ \forall & \text { Para todo } \\ \exists & \text { Existe } \\ \mathbb{Q} & \text { Conjunto de números racionales } \\ \mathbb{R} & \text { Conjunto de números reales } \\ \wedge & \text { Conjunción lógica } \\ \vee & \text { Disyunción lógica } \\ \rightarrow & \text { Condicional material } \\ \leftrightarrow & \text { Bicondicional } \\ () & \text { Paréntesis } \\ \therefore & \text { Por lo tanto }\end{array}$

Juan de Dios González Ibarra Posdoctorado en Filosofía de las Ciencias Sociales y Humanidades UPV, España, Licenciado en Derecho y Administración, Doctor en Ciencias Políticas y Sociales, pasante de Doctor en Economía UNAM. PTC UAEM, SNI II CONACYT, México.E-mail: drdiosgi@hotmail.com. Endereço profissional: Privada Margaritas 18, Col. Lomas de Cortés. Cuernavaca, Morelos, México. C. P. 62240.

José Luis Díaz Salazar Licenciado y Maestro en Derecho UAEM, Pasante de Doctor en Ciencias Políticas CIDHEM, México.E-mail: joseluisdiazsalazar@ gmail.com.

Endereço profissional: Miguel Hidalgo Poniente, 1047, Toluca, México. Código Postal 50080. 